\title{
DOPPLER MODEL FOR ADIABATIC TOROIDAL COMPRESSOR DRIFTWAVE FREQUENCY PROFILES
}

\section{PLASMA PHYSICS LABORATORY}

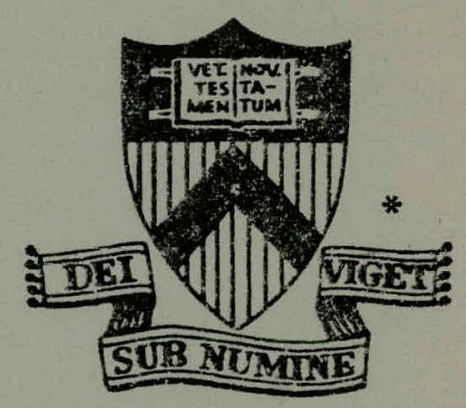

\section{PRINCETON UNIVERSITY PRINCETON, NEW JERSEY}

This work was supported by U. S. Energy Research and Development Administration Contract EY-76-C-02-3073. Reproduction, translation, publication, use and disposal, in whole or in part, by or for the United States Government is permitted. 


\section{DISCLAIMER}

This report was prepared as an account of work sponsored by an agency of the United States Government. Neither the United States Government nor any agency Thereof, nor any of their employees, makes any warranty, express or implied, or assumes any legal liability or responsibility for the accuracy, completeness, or usefulness of any information, apparatus, product, or process disclosed, or represents that its use would not infringe privately owned rights. Reference herein to any specific commercial product, process, or service by trade name, trademark, manufacturer, or otherwise does not necessarily constitute or imply its endorsement, recommendation, or favoring by the United States Government or any agency thereof. The views and opinions of authors expressed herein do not necessarily state or reflect those of the United States Government or any agency thereof. 


\section{DISCLAIMER}

Portions of this document may be illegible in electronic image products. Images are produced from the best available original document. 


\section{NOTICE}

This report was prepared as an account of work sponsored by the United States Government. Neither the United States nor the United States Energy Research and Development Administration, nor any of their employees, nor any of their contractors, subcontractors, or their employees, makes any warranty, express or implied, or assumes any legal liability or responsibility for the accuracy, completeness or usefulness of any information, apparatus, product or process disclosed, or represents that its use would not infringe privately owned rights.

Printed in the United states of America.

Available from

Niational Technical Information Service

U. S. Department of Commerce 5285 Port Royal Road

Springfield, Virginia $22 \mathrm{~J} 51$

price: Printed Copy $\$$ *; Microfiche $\$ 3.00$

$$
\begin{gathered}
\text { *Pages } \\
\begin{array}{c}
1-50 \\
51-150 \\
151-325 \\
326-500 \\
501-1000
\end{array}
\end{gathered}
$$

NTIS

Selling Price

$\$ 4.00$

5.45

7.60

10.60

13.60 
Doppler Model for Adiabatic Toroidal Compressor

Driftwave Frequency Profiles

by

R. A. Koch and W. M. Tang

Princeton University, Plasma Physics Laboratory

Princeton, New Jersey 08540

PPPI-1381

October 1977

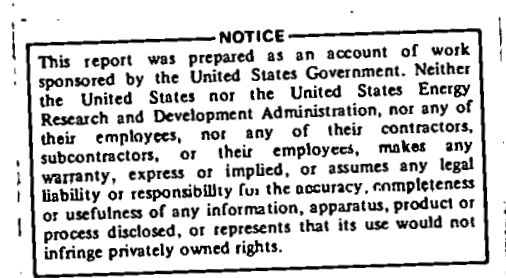


Doppler Model for Adiabatic Toroidal Compressor Driftwave Frequency Profiles

R. A. Koch and W. M. Tang

Princeton University, Plasma Physics Laboratory

Princeton, New Jersey 08540

Microwave and $\mathrm{CO}_{2}$ laser scattering experiments on ATC (Adiabatic Toroidal Compressor) Tokamak have shown that there exists a spectrum of fluctuations with $|f|=|\omega / 2 \pi| \lesssim 1 \mathrm{MHz}$ at wavelengths $\lambda \sim 0.28 \mathrm{~cm}$ and with $|\mathrm{f}| \leqq 400 \mathrm{kHz}$ for $\lambda \sim 1 \mathrm{~cm}$. However, a typical maximum frequency calculated for given experimental density and temperature profiles is $\mathrm{f} \leqslant 150 \mathrm{kHz}$ for the longer wavelengths and differs with the experimental results by roughly a factor of 2 or 3 . At shorter wavelengths the calculated maximum $f \leqslant 100 \mathrm{kHz}$, which differs quite substantially with the experimental result. We interpret this discrepancy as evidence of an $\vec{E} \times \vec{B} \cdot$ Doppler shift from a radial electric field. 
Fluctuation spectra associated with drift waves have been observed on ATC Tokamak using microwave ${ }^{1}$ and $\mathrm{CO}_{2}$ laser ${ }^{2}$ scattering techniques. In the microwave scattering experiment, fluctuations with wavelengths $0.7 \mathrm{~cm} 5 \lambda \leqslant 1.0 \mathrm{~cm}$ were found to have spectral components with maximum frequencies, $f$, in the range $300 \mathrm{kHz} \leqslant f=(\omega / 2 \pi) \lesssim 400 \mathrm{kHz}$. In the same experiment the frequencies were observed to change sign, while the spectrum maintains the same shape, though inverted, when the toroidal magnetic field direction is changed. In the laser scattering experiment, fluctuations with wavelengths $0.28 \mathrm{~cm} \leq \lambda \leq 2 \mathrm{~cm}$ were observed. At longer wavelengths the maximum frequency is in the same range as in the microwave experiment, but can be as large as $1 \mathrm{MHz}$ at the shorter wavelengths.

For a tokamak plasma of electrons and singly-charged ions the electron and ion collisionality are given by

$$
\begin{aligned}
& \nu_{* e}=\frac{4(2 \pi)^{1 / 2}}{3} \frac{r B_{0}}{B_{p}}\left(\frac{R}{r}\right)^{3 / 2} \frac{\mathrm{n}_{i} e^{4} \ln \Lambda}{T_{e}^{2}} \\
& \nu_{* i}=2^{-1 / 2}\left(\mathrm{~T}_{e} / \mathrm{T}_{\mathrm{i}}\right)^{2} \nu_{* e}
\end{aligned}
$$

where $R$ is the distance to the major axis, $r$ is the distance to the minor axis, $B_{0}=\bar{B}_{T}(r=0)$ is the toroidal magnetic field at $r=0, B_{p}$ is the poloidal magnetic field, $n_{i}$ is the ion density, $T_{e}\left(T_{i}\right)$ is the electron (ion) temperature, and the Coulomb logarithm is 


$$
23.4-1.15 \cdot \log _{10} n_{e}+3.45 \log _{10} T_{e} \quad T_{e}<50 \text { eV }
$$

$\ln \Lambda=$

$$
25.3-1.15 \log _{10} \mathrm{n}_{\mathrm{e}}+2.3 \log _{10} \mathrm{~T}_{\mathrm{e}} \quad \mathrm{T}_{\mathrm{e}}>50 \mathrm{eV}
$$

For $\nu_{* e} \geq 0.5$ characteristic of the experiment, dissipative trapped-electron and collisionless drift modes should be present over a large part of the minor radius. The scattering volumes in both the microwave and $\mathrm{CO}_{2}$ laser experiments also intersect the plasma over a range of minor radii and poloidal angles depending on the plasma region selected for observation. Hence, it would be expected that the observed spectra should contain a range of frequencies and amplitudes for given $\lambda$ corresponding to the variation of the density and temperature profiles and of the scattering angle over the scattering volume. We have written a simple computer code which calculates, using profiles 3 typical of the se experiments, the radial variation of $f_{D W}=\omega_{D W} / 2 \pi$, where

$$
\begin{aligned}
& \omega_{\mathrm{DW}}=\omega_{* \mathrm{e}} \mathrm{S} \\
& \omega_{* \mathrm{e}}=-\frac{\mathrm{ck}{ }_{\perp} \mathrm{T}_{\mathrm{e}}}{\mathrm{eB} \mathrm{B}} \frac{1}{\mathrm{n}_{\mathrm{e}}} \frac{\mathrm{dn} \mathrm{e}_{\mathrm{e}}}{\mathrm{dr}} \\
& \mathrm{S}=\frac{\Gamma_{\mathrm{o}}+\eta_{\mathrm{i}} \mathrm{b}\left(\Gamma_{1}-\Gamma_{\mathrm{o}}\right)}{1+\tau\left(1-\Gamma_{0}\right)} \\
& \mathrm{b}=\left(\mathrm{k}_{\perp} \rho_{\mathrm{i}}\right)^{2} / 2 \\
& \rho_{\mathrm{i}}=\frac{\mathrm{v}_{\mathrm{i}}}{\Omega_{\mathrm{i}}}=\left(\frac{2 \mathrm{~T}_{\mathrm{i}}}{\mathrm{m}_{\mathrm{i}}}\right)^{1 / 2} /\left(\frac{\mathrm{eB} \mathrm{T}}{\mathrm{m}_{\mathrm{i}} \mathrm{c}}\right)
\end{aligned}
$$




$$
\begin{aligned}
& \Gamma_{0,1}=e^{-b} I_{0, l}(b) \\
& \eta_{i, e}=\frac{d \ln T_{i, e}}{d \ln n_{i, e}} \\
& \tau=T_{e} / T_{i}
\end{aligned}
$$

and $\mathrm{I}_{0,1}$ are modified Bessel functions. In this calculation we take $k_{1}=$ $k=2 \pi / \lambda$, although we note that generally ${ }^{1,2} k_{\perp} \leqslant k$ because the re will be a finite $k_{\|}$and $k_{r}, k_{\theta}$ decomposition associated with the fluctuations. The maximum value of $f$ obtained from this calculation for $\lambda \sim 1 \mathrm{~cm}$ was less than $150 \mathrm{kHz}$ with $\mathrm{k}_{\perp} \rho_{\mathrm{i}} \lesssim 1$ over most of the cross section. For $\lambda \sim 0.28 \mathrm{~cm} \max \left\{f_{D W}\right\} \leqslant 100 \mathrm{kHz}$ with $\mathrm{k}_{\perp} \rho_{i} \geq 1$ over most of the cross section. Typical profile values at $r=0$ for these calculations were

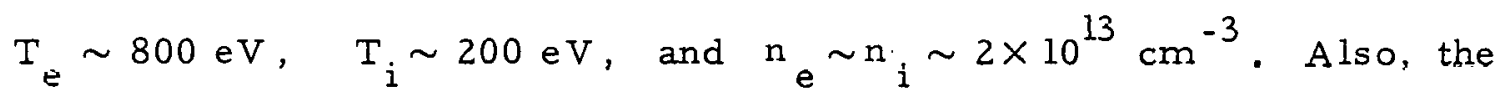
generally broader ion temperature profiles also allowed $0.2 \leq \tau \leq 5.0$. It appears that the maximum frequency observed for the fluctuations is 2 - 10 times the maximum value calculated using Eq. (1) for the given experimental profiles. In particular, this discrepancy is quite pronounced at shorter wavelengths.

To explain the discrepancy we consider the possibility of the re being a Doppler shift included in the observed frequency. Such an effect has been observed $^{4-6}$ previously in a variety of experiments. Thus, we take ${ }^{7}$ for the observed frequency 


$$
\omega=\omega_{\mathrm{DW}}+\omega_{\mathrm{DOPP}}
$$

with

$$
\omega_{\text {DOPP }}=\frac{c k}{B_{T}} \frac{d \Phi}{d r}
$$

where $\Phi$ is the lowest order electrostatic potential. In order to explain the observed frequency spectrum this model $\omega_{\text {DOPP }}$ must also have radial variation on the same scale as $\omega_{\mathrm{DW}}$. The value of $d \Phi / d r$ was not measured directly for the ATC Tokamak and must be calculated. According to neoclassical theory the time asymptotic value of this field depends upon the fastest nonambipolar diffusion process. In a completely ionized electron and single ion species plasma, neoclassical diffusion is ambipolar, and the steady state potential is determined at higher order in the gyroradius. This problem has been considered in the banana 8 and Pfirsch-Schlüter ${ }^{9}$ limits. Neither of these is appropriate because in the ATC Tokamak the ions are a predominately plateau regime species and because the time scale on which the potential is established is much longer than the duration of the experiment. Thus, we consider nonambipolar processes capable of establishing the potential on shorter time scales. For ORMAK (Oak Ridge Tokamak Device) it has been proposed that one such process is charge exchange. ${ }^{10}$ For the ATC Tokamak the same conclusion appears to be valid. ${ }^{11}$ The potential is determined by the condition that there is no ion toroidal momentum on any magnetic surface. The basis for this condition is that in the steady state there is no net momentum transfer between ions 
and neutrals via charge exchange while the neutral particles transfer all of their momentum to the walls. The radial ion flux driven by any net ionneutral momentum exchange has no electron counterpart and thereby provides the freedom for the nonambipolar change in the potential necessary to satisfy the condition on the ion toroidal momentum. Thus, for $t \gg \nu_{\mathrm{cx}}^{-1}$, the potential is then determined ${ }^{10}$ by

$$
0=R_{\| c x} \propto u_{\| i}+ \begin{cases}O V\left(\frac{r}{R}\right) & \text { banana } \\ O\left(\frac{r}{R}\right) & \text { plateau }\end{cases}
$$

where ${ }^{12}$

$$
u_{i \|} \simeq \frac{c T_{i}}{e B_{p}}\left[\left(\frac{5}{2}-y\right) \frac{d \ln T_{i}}{d r}-\frac{d \ln p_{i}}{d r}-\frac{e}{T_{i}} \frac{d \Phi}{d r}\right]
$$

with

$$
\frac{5}{2}-y=\frac{1.17-0.35 \nu_{* i}^{1 / 2}}{1+0.7 \nu_{* i}^{1 / 2}} .
$$

Thus, to lowest order in the small aspect ratio limit,

$$
\omega_{\mathrm{DOPP}} \approx \tau \omega_{* e}\left\{1+\eta_{\mathrm{i}}[1-(5 / 2-\mathrm{y})]\right\},
$$

and in the plateau regime

$$
\frac{\omega_{\text {DOPP }}}{\omega_{D W}} \approx \frac{\tau\left[1+3 / 2 \eta_{i}\right]}{S} .
$$


For $\eta \sim \tau \sim 1$ we obtain $\omega_{\text {DOPP }} / \omega_{D W} \sim 10$ when $k_{\perp} \rho_{i} \sim 1$. When the experimental profiles are used to calculate $\max \left[f=\left(\omega_{D W}+\omega_{D P P}\right) / 2 \pi\right]$ we obtain values $\sim 500 \mathrm{kHz}$ for $\lambda=1 \mathrm{~cm}$ and $\sim 1.3 \mathrm{MHz}$ for $\lambda \sim 0.28 \mathrm{~cm}$ in reasonable agreement with the scattering results.

We consider now the applicability of our model to the fluctuation spectra for other experiments. First, microwave scattering on TFR ${ }^{13}$ (FontenayAux-Roses Tokamak) indicates $f=(\omega / 2 \pi) \sim(3-5)\left(\omega_{\mathrm{DW}} / 2 \pi\right)$. The Doppler shifted frequencies of our model are in quantitative agreement with this result. How ever, for the PLT Tokamak the maximum frequencies observed appear to be above $\max \left(\omega_{D W} / 2 \pi\right)$, but below $\max \left[\left(\omega_{D W}+\omega_{D O P P}\right) / 2 \pi\right]$. This result may be a consequence of the fact that the neutral particle mean free paths are shorter than the PLT Tokamak minor radius ${ }^{15}$ so that $u_{i \| l}=0$ is no longer a good approximation. In other words, momentum transfer from ions to neutrals via charge exchange does not imply $u_{i \| l}=0$ unless the neutrals also lose momentum, e.g., to the walls. The solution for $u_{i l l}$ in this regime remains open to investigation. For ALCATOR (Massachusetts Institute of Technology Tokamak), we expect at lower densities results similar to those in the ATC Tukamak. At higher densities in ALCATOR, a situation similar to that in PLT Tokamak regarding the neutral particle mean free path may arise. A possible complicating factor in the latter regime is the field ripple associated with diagnostic ports un ALCATOR. The ripples may be sufficiently large to produce non-ambipolar diffusion ${ }^{16}$ without charge exchange effects and give qualitatively the same results for $\omega_{\text {DOPP }}$ as discussed above. 
At this point we note that it has recently been proposed ${ }^{17}$ that nonlinear effects could account for the observed frequency spectrum. The result is a frequency spread around $\omega=0$ proportional to $k^{3} /\left(1+k^{2}\right)$, where $k$ is normalized by $c_{s} / \Omega_{i}$. This theory. requires for its validity the existence of a $k=0$ potential with $e \phi / T_{e} \sim 0.2$ to give the observed frequency spread. If both exist, the nonlinear effect and the Doppler shift would presumably be additive. This means that the $k \approx 0$ potential necd not be as large in order to give the observed spread. It should also be noted that this nonlinear theory ${ }^{17}$ seems to predict spectra symmetric about $\omega=0$ and hence invariant when $\vec{B}$ changes direction. This, however, is in contradiction with the experimental observation. 1

In conclusion the Doppler shifted drift wave frequency model appears to account for the frequencies $\omega>\omega_{\mathrm{DW}}$ observed in ATC. With regard to the nonlinear theory of drift waves our result provides support for the weak turbulence approach. In particular, our calculation as sumes that the observed frequencies are approximately the mode frequencies of linear theory (though Doppler shifted) consistent with a basic requirement for the validity of weak turbulence theory. We note that the observed ${ }^{1,2}$ relative fluctuation amplitudes $|\tilde{n} / \bar{n}| \sim 0.03$ are also consistent with the weak turbulence assumption. However, this fact alone would be insufficient basis to invoke a weak turbulence description if substantial nonlinear broadening of the mode frequencies, associated with strong turbulence, was necessary to account for the observation of frequencies with $\omega>\omega_{D W} \cdot$ 


\section{ACKNOW LEDGMENTS}

We wish to thank Drs. E. Mazzucato, C. M. Surko, R. E. Slusher, S. Suckewer, and H. Hsuan for their cooperation and discussions concerning the ATC experiment. We also acknowledge enlightening discussions with Drs. R. J. Goldston, R. D. Hazeltine, W. Horton, S. P. Hirshman, P. H. Rutherford, and E. A. Frieman. Finally, we wish to remark that Dr. C. Oberman has also suggested that a Doppler effect might account for the experimentally observed frequency spread.

Work supported by U.S. Energy Research and Development Administration Contract no. EY-76-C-02-2073. 


\section{REFERENCES}

${ }^{1}$ E. Mazzucato, Phys. Rev. Lett. 36, 792 (1976).

${ }^{2}$ C. M. Surko and R. E. Slusher, Phys. Rev. Lett. 37, 1747 (1976).

${ }^{3}$ Electron density and temperature profiles were supplied by $E$. Mazzucato, and by R. E. Slusher and C. M. Surko for their experiments respectively. Ion temperature profiles were taken from S. Suckewer and E. Hinnov, Frinceton Plasma Physics Laburatury Report PPPL=1323 (1977), and from R. J. Goldston, E. Mazzucato, R. E. Slusher, and C. M. Surko, PPPL-1290 (1976).

${ }^{4}$ P. Brossier, P. Deschamps, R. Gravier, R. Hellat, and $C$. Renaud, Phys. Rev. Lett. 26, 124 (1971).

${ }^{5}$ J. C. Hosea, F. C. Jobes, R. L. Hickok, and A. N. Dellis, Phys. Rev. Lett. 30, 839 (1973).

${ }^{6}$ R. F. Ellis and R. W. Motley, Phys. Fluids 17, 582 (1974).

7 L. Cheung and W. Horton, Annals of Physics 81, 201 (1973).

8 M. N. Rosenhluth, P. H. Rutherford. J. B. Taylor, E. A. Frieman, L. M. Kovrizhnikh, Plasma Physics and Controlled IVuclear Fusion Resedrch (International A tomic Energy Agency, Vienna, 1971), Vol. 1, p. 495.

${ }^{9}$ R. D. Hazeltine, Phys, Fluids 17, 961 (1974).

10 D. J. Sigmar, J. F. Clarke, R. V. Neidigh, and K. L. Vander Sluis, Phys. Rev. Lett. 33, 1376 (1974). 
${ }^{11}$ R. J. Goldston, private communication.

${ }^{12}$ F. L. Hinton and R. D. Hazeltine, Rev. Mod. Phys. 48, 239 (1976).

${ }^{13}$ T.F.R. Group, Plasma Physics and Controlled Nuclear Fusion Research (Proc. 6th International Conference Berchtesgaden, 1976), Vol. I, p. 35, (I. A. E. A. Vienna, 1977).

${ }^{14}$ E. Mazzucato, private communication.

${ }^{15}$ M. H. Hughes and D. E. Post, Princeton Plasma Physics Laboratory PPPL-1335 (1977).

${ }^{16}$ K. T. Tsang, Ph. D. Dissertation (unpublished) Princeton University 1974; K. T. Tsang and E. A. Frieman, Phys. Fluids 19, 747 (1976).

17 A. Hasegawa and K. Mima, Phys. Rev. Lett. 39, 205 (1977). 